\title{
CIE JOURNAL/REVUE ECI 2010 REFEREES/EVALUATEURS
}

We would like to thank the many people who contributed their time and expertise to review articles for Volume 39 of the Canadian and International Education Journal.

Nous voudrions remercier toutes les personnes qui ont contribué leur temps et leur expertise pour évaluer les articles soumis à la Revue d'éducation canadienne et internationale Numéro 39.

\section{Paul Carr}

Rethy Chhem

Julie Byrd Clark

Adam Davidson-Harden

Colette Despagne

Cecille DePass

Peter Fan

Dawn Fyn

Kelly Crowley Thorogood

Kathy Hibbert

Marianne Larsen

Xuemei Li

Mirela Moldoveanu

Immaculate Namukasa

Jenny Newton

Deo Poonwassie

David Radcliffe

Kara Smith

Marilyn Steinbach

Roz Stooke

Paul Tarc

Shelley Taylor

Teresa Van Deven

Aniko Varpalotai

Bing Wang

Zheng Zhang 